This item was submitted to Loughborough's Research Repository by the author.

Items in Figshare are protected by copyright, with all rights reserved, unless otherwise indicated.

\title{
Improved accuracy in quantitative fault tree analysis
}

PLEASE CITE THE PUBLISHED VERSION

PUBLISHER

(c) The authors

LICENCE

CC BY-NC-ND 4.0

REPOSITORY RECORD

Sinnamon, Roslyn M., and J.D. Andrews. 2008. "Improved Accuracy in Quantitative Fault Tree Analysis". figshare. https://hdl.handle.net/2134/3728. 
This item was submitted to Loughborough's Institutional Repository by the author and is made available under the following Creative Commons Licence conditions.

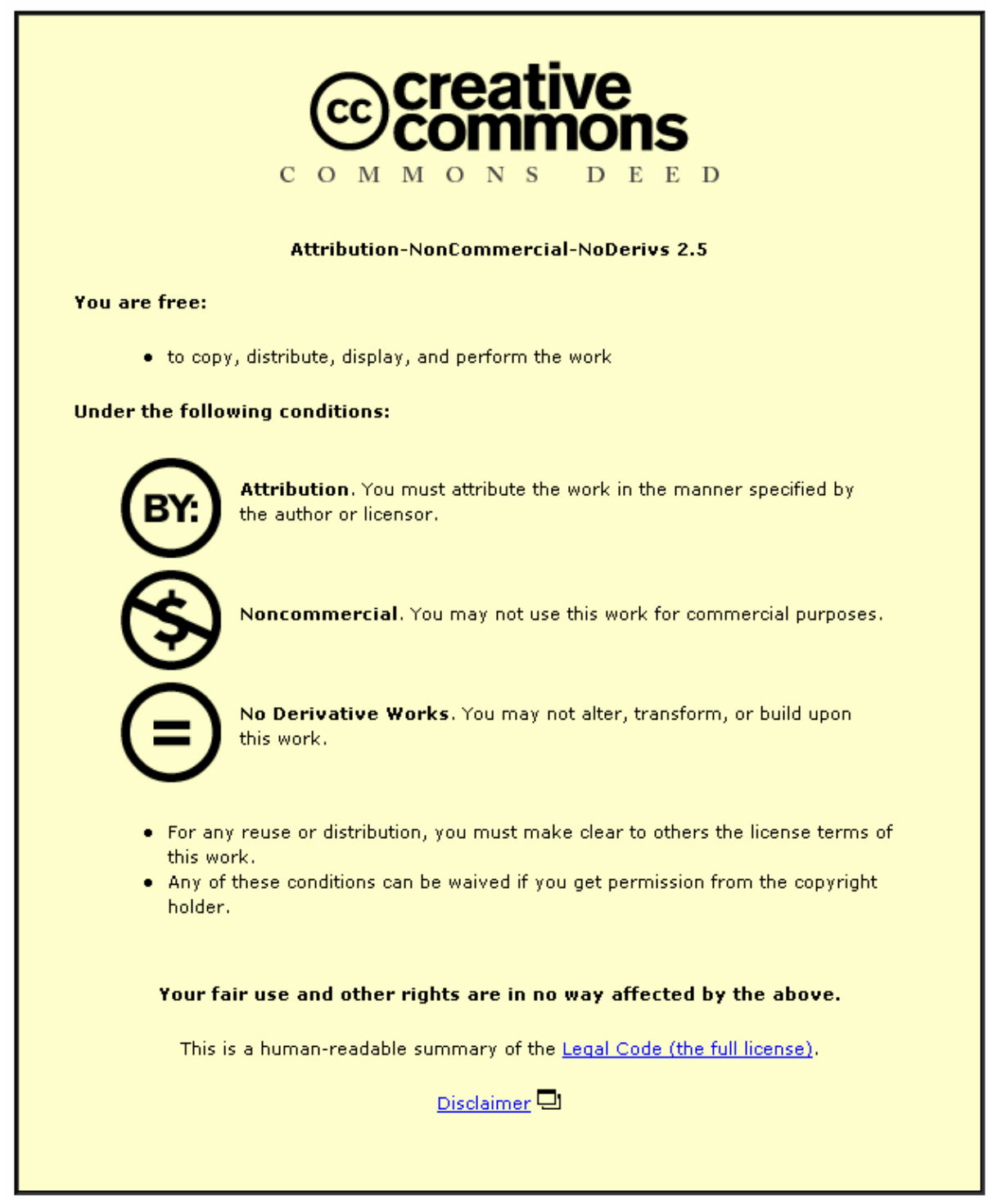

For the full text of this licence, please go to: http://creativecommons.org/licenses/by-nc-nd/2.5/ 


\title{
Improved Accuracy in Quantitative Fault Tree Analysis
}

\author{
R M Sinnamon and J D Andrews \\ Department of Mathematical Sciences \\ Loughborough University of Technology \\ Loughborough, Leics LE11 3TU.
}

\section{Summary}

The fault tree diagram defines the causes of the system failure mode or "top event" in terms of the component failures and human errors, represented by basic events. By providing information which enables the basic events' probability to be calculated the fault tree can then be quantified to yield reliability parameters for the system.

Fault tree quantification enables the probability of the top event to be calculated and in addition its failure rate and expected number of occurrences. Importance measures, which signify the contribution each basic event makes to system failure can also be determined. Due to the large number of failure combinations (minimal cut sets) which generally result from a fault tree study, it is not possible using conventional techniques to calculate these parameters exactly and approximations are required. The approximations usually rely on the basic events having a small likelihood of occurrence. When this condition is not met it can result in large inaccuracies. These problems can be overcome by employing the Binary Decision Diagram approach. This method converts the fault tree diagram into a format which encodes Shannon's decomposition and allows the exact failure probability to be determined in a very efficient calculation procedure.

This paper describes how the Binary Decision Diagram method can be employed in fault tree quantification.

\section{Introduction}

The Binary Decision Diagram (BDD) method, developed by Rauzy (Ref. 1), converts the fault tree to a binary decision diagram which encodes an If-Then-Else (ite) structure. An attractive feature of the BDD method is that the ite structure derives from Shannon's formula (Ref 2), such that if $f(x)$ is the Boolean function for the fault tree top event then by pivoting about any variable $\mathrm{X} 1$ the Shannon formula can be written as:

$$
X 1 . f 1+\overline{X 1} \cdot f 2
$$


where $f 1$ and $f 2$ are Boolean functions with $X 1=1$ and $X 1=0$ respectively which are of one order less than $f$. The corresponding ite structure is ite $(X 1, f 1, f 2)$. A detailed account of this procedure is given in Ref 3 and Ref 4 .

The size of the resulting BDD is very dependent upon the ordering chosen for the basic event pivot variables in the fault tree. Usually a Top-Down ordering is used, where the basic events occurring higher up the tree are considered as being less than those which occur lower down. This ordering of variables is discussed in Ref. 3 and covered in more detail in the paper, "Improved Efficiency in Qualitative Fault Tree Analysis" (Ref 5).

\section{Example}

Consider the simple fault tree illustrated in figure 1.

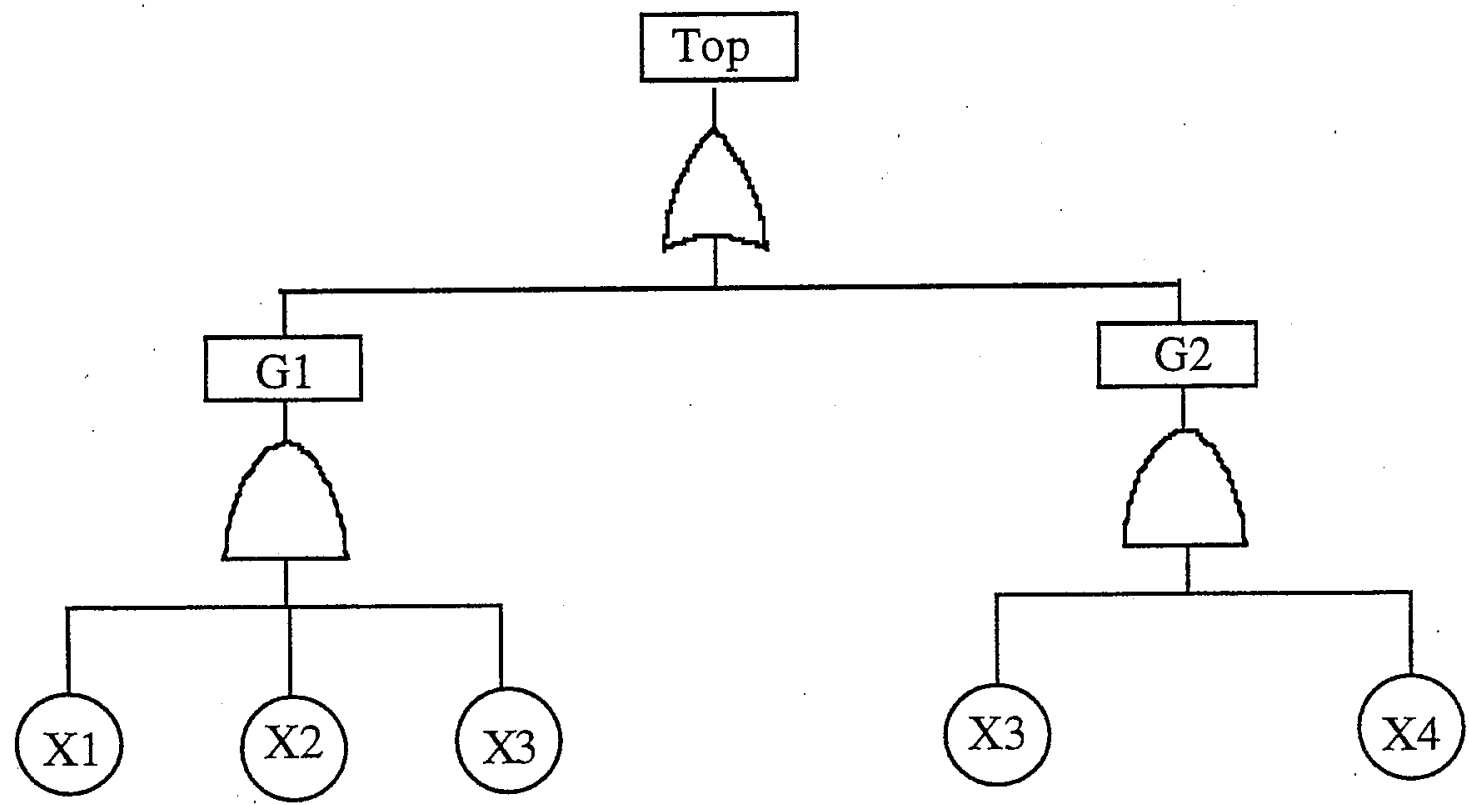

Figure 1. Example Fault Tree

The minimal cut sets for this fault tree are:

(1) $\{X 1, X 2, X 3\}$

(2) $\{X 3, X 4\}$

Therefore, its structure function, $\phi(\mathbf{x})$ is :

$$
\phi(\mathbf{x})=1-(1-\mathrm{X} 1 . \mathrm{X} 2 . \mathrm{X} 3)(1-\mathrm{X} 3 . \mathrm{X} 4)
$$

A Top-Down ordering of basic events would give; 
since all basic events are on the same level. This ordering will yield the BDD shown in figure 2 (figure $2 \mathrm{a}$ with the Boolean equations to show its development from the structure function and the simplified form in figure $2 b$ ).

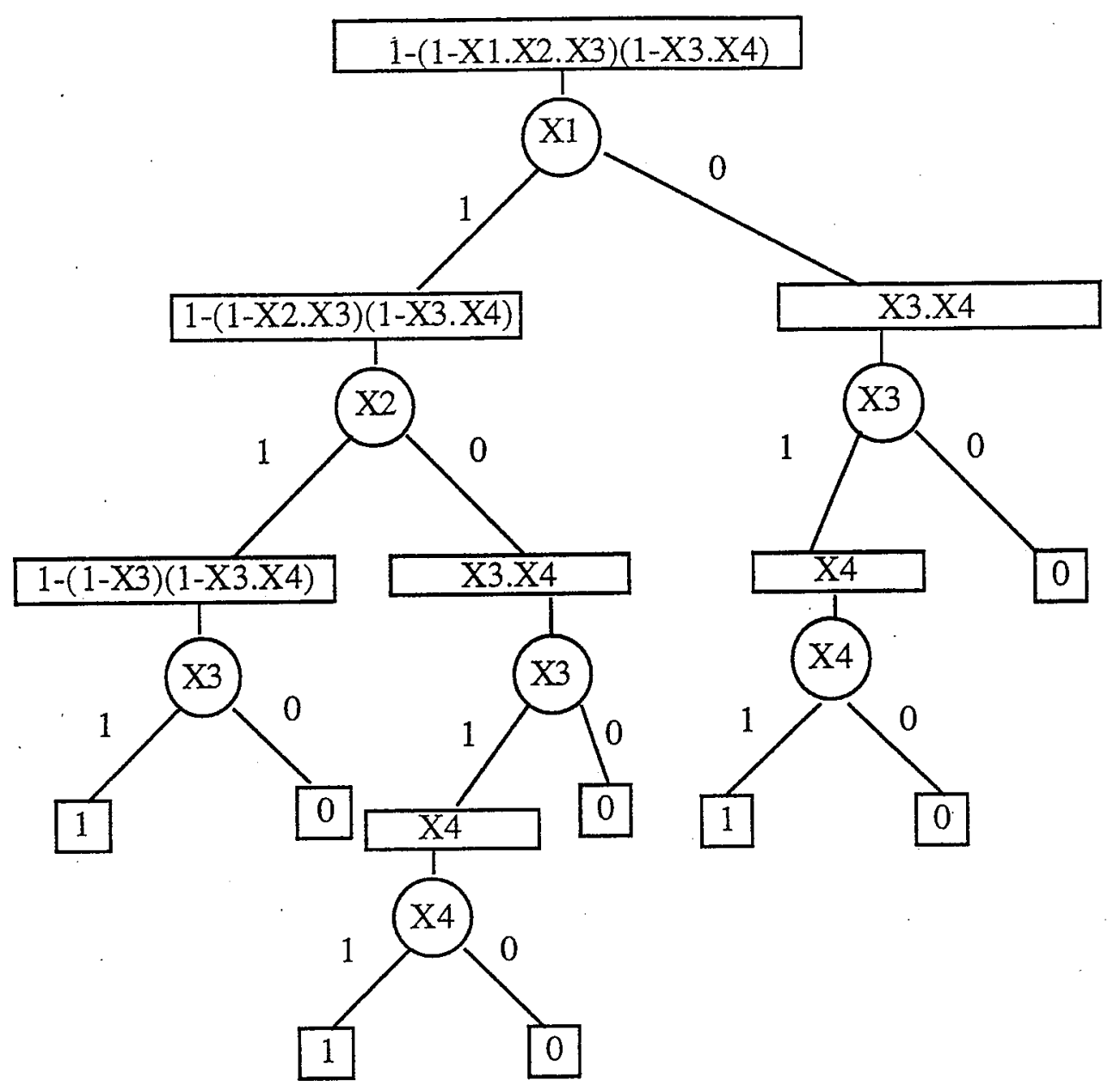

Figure 2a. BDD with Boolean Equations 


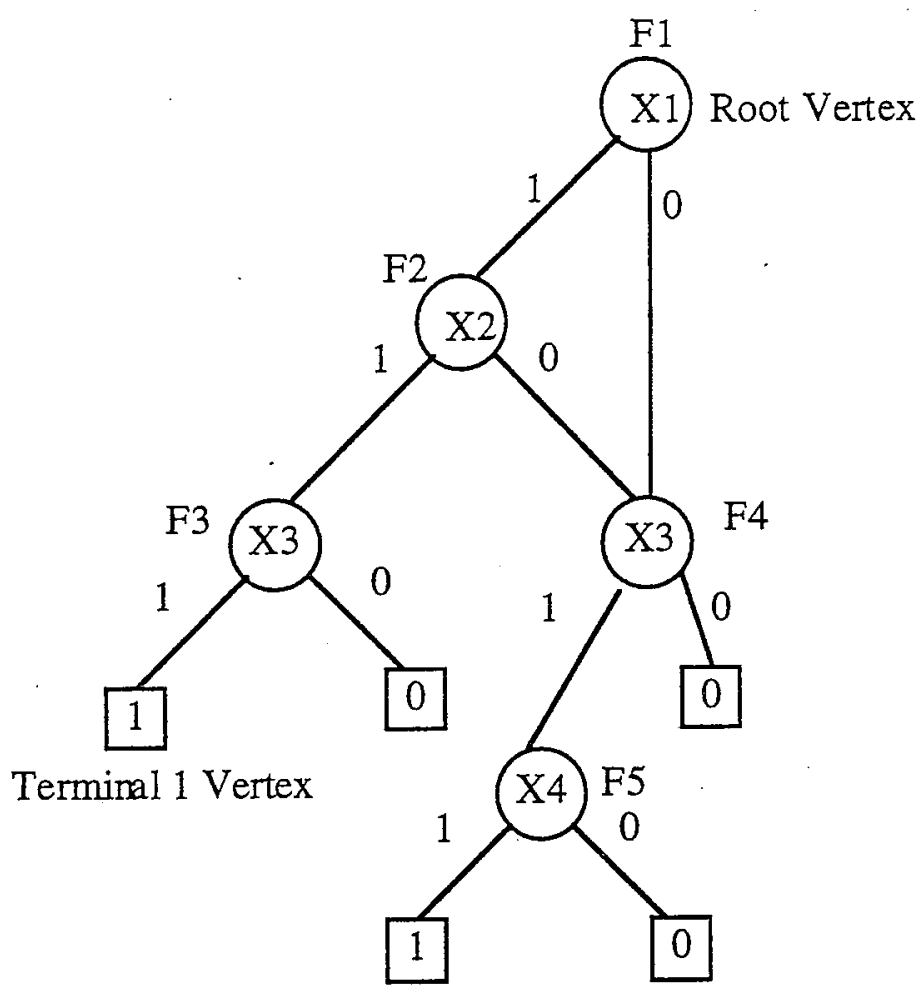

Figure $2 b . \mathrm{BDD}$ for the fault tree shown in figure 1

To obtain the cut sets of the fault tree paths are traced through the BDD from the top or root vertex to a terminal 1 vertex. Only the basic events that lie on a 1 branch (indicating the failure of that basic event) for each component are included in the cut set. Therefore the cut sets of the $B D D$ shown in figure $2 b$ are:

(1) $\mathrm{X} 1 . \mathrm{X} 2 . \mathrm{X} 3$

(2) $\mathrm{X} 1 . \mathrm{X} 3 . \mathrm{X} 4$

(3) $\mathrm{X} 3 . \mathrm{X} 4$

The resulting BDD for this basic event ordering is not minimum, as it produces one redundant cut set. To obtain the minimal cut sets either the resulting cut sets can be reduced or the BDD can undergo a minimising procedure, whose details are given in Ref. 3. However for the purposes of quantification, the non-minimal BDD is used.

\section{Top Event Probability}

The conventional approach (see Andrews and Moss Ref (6), Henley and Kumamoto Ref (7)) to obtain the exact probability of the top event is to use the formula:

$P(T o p)=\sum_{i=1}^{n c} P\left(C_{i}\right)-\sum_{i=2}^{n c} \sum_{j=1}^{i-1} P\left(C_{i} \cap C_{j}\right)+\ldots \ldots . . \ldots \ldots . .(-1)^{n c-1} P\left(C_{1} \cap C_{2} \cap \ldots \ldots . \cap C_{n c}\right)$ 
Where $C_{i}, \mathrm{i}=1, \ldots \ldots \ldots . . \mathrm{nc}$ are the minimal cut sets of the top event.

Clearly if the fault tree has many minimal cut sets, calculating $\mathrm{P}(\mathrm{Top})$ will require extensive calculations to evaluate each term in expression (4). For many complex fault trees this processing requirement is beyond the capability of the available machines. In order to reduce the calculations to a practical size approximations can be used. The Rare Event Approximation, $P_{R E}(T o p)$, is commonly used:

$$
P_{R E}(T o p)=\sum_{i=1}^{n c} P\left(C_{i}\right)
$$

However a more accurate approximation is the Minimal Cut Set Upper Bound:

$$
P_{M C S U B}(T o p)=1-\prod_{i=1}^{n c}\left(1-P\left(C_{i}\right)\right)
$$

The Binary Decision Diagram method, avoids the need to utilise these approximations as the probability of the top event is obtained directly from the diagram.

Since the ite structure for the top event of a fault tree is derived from Shannons' formula, i.e., if $f(x)=i t e(X 1, f 1, f 2)$ then the corresponding Boolean function or structure function is $f(\mathbf{x})=X 1 . f 1+\bar{X} 1 . f 2$. When a Boolean function is expressed in this form the probability of the top event is obtained by taking the expectation of each term:

$$
\mathrm{E}[\mathrm{f}(\mathbf{x})]=q_{1} \cdot \mathrm{E}[\mathrm{f} 1]+\left(1-q_{1}\right) \cdot \mathrm{E}[\mathrm{f} 2]
$$

where $q_{1}=\mathrm{E}[\mathrm{X} 1]$, the probability that event 1 has occurred.

Therefore the probability of occurrence of the top event $\left(Q_{s y s}\right)$, can be expressed as the sum of the probabilities of the disjoint paths through the BDD. The disjoint paths through the BDD are found by simply including in a path from the root vertex to a terminal 1 node, all events which lie on the 1 branch and the 0 branches for each the basic events. Basic events which lie on a 0 branch are indicating in the paths as $\overline{X i}$. Disjoint paths through the BDD shown in figure $2 \mathrm{~b}$ are:

(1) $\mathrm{X} 1 . \mathrm{X} 2 . \mathrm{X} 3$

(2) $\mathrm{X} 1 . \overline{\mathrm{X} 2} \cdot \mathrm{X} 3 . \mathrm{X} 4$

(3) $\overline{X 1} . X 3 . X 4$ 
Before performing with the calculation of $\mathrm{Q}_{\text {sys }}$ the basic events in the fault tree need to be assigned probabilities. For the fault tree shown in Figure 1 the component data provided on table 1 will be used.

\begin{tabular}{|c|c|c|c|}
\hline basic event $\mathbf{i}$ & $\mathbf{q}_{\mathbf{i}}$ & $\lambda_{\mathbf{i}}$ & $\mathbf{w}_{\mathbf{i}}=\lambda_{\mathbf{i}}\left(\mathbf{1}-\mathbf{q}_{\mathbf{i}}\right)$ \\
\hline $\mathrm{X} 1$ & 0.004 & $1.2 \mathrm{E}-6$ & $4.8 \mathrm{E}-9$ \\
\hline $\mathrm{X} 2$ & 0.003 & $4.3 \mathrm{E}-6$ & $1.29 \mathrm{E}-8$ \\
\hline $\mathrm{X} 3$ & 0.002 & $2.5 \mathrm{E}-6$ & $5.0 \mathrm{E}-9$ \\
\hline $\mathrm{X} 4$ & 0.001 & $3.1 \mathrm{E}-6$ & $3.1 \mathrm{E}-9$ \\
\hline
\end{tabular}

Table 1. Basic Event Data.

In table $1 \mathbf{q}_{\mathbf{i}}$ is the Unavailability of component $i, \lambda_{\mathbf{i}}$ is the Conditional failure intensity of component $i$, and $\mathbf{w}_{\mathbf{i}}$ is the Unconditional failure intensity of component $i$.

Using this data the top even probability $\mathrm{Q}_{\text {sys }}$ can be calculated as follows;

$$
\begin{aligned}
-Q_{\text {sys }} & =P(X 1 \cdot X 2 \cdot X 3+X 1 \cdot \overline{X 2} \cdot X 3 \cdot X 4+\overline{X 1} \cdot X 3 \cdot X 4) \\
& =q_{X 1} \cdot q_{X 2} \cdot q_{X 3}+q_{X 1} \cdot\left(1-q_{X 2}\right) \cdot q_{X 3} \cdot q_{X 4}+\left(1-q_{X 1}\right) \cdot q_{X 3} \cdot q_{X 4} \\
& =2.023976 \mathrm{E}-6
\end{aligned}
$$

\section{Unconditional System Failure Intensity}

For some systems it is required to calculate the unreliability for the top event i.e., the probability it will not work continuously over a given time period. An upper bound for this is the expected number of top event occurrences, $W(0, t)$ :

$$
W(0, t)=\int_{0}^{t} w_{\text {sys }} d t
$$

where $w_{\text {sys }}$ is the system unconditional failure intensity. This can be expressed as:

$$
w_{\text {sys }}=\sum_{i} G_{i}(\mathbf{4}) \cdot w_{i}
$$

where $G_{i}(\mathbf{q})$ is the criticality function for each component. 
The criticality function $G_{i}(\mathbf{q})$ is defined as the probability that the system is in a critical state with respect to component $i$ and that the failure of component $i$ will then cause the system to go from the working state to the failed state, i.e., the probability that the system fails only if component i fails. Therefore:

$$
G_{i}(\mathbf{q})=Q\left(1_{i}, \mathbf{q}\right)-Q\left(0_{i}, \mathbf{q}\right)
$$

Where:

$Q\left(1_{i}, \mathbf{q}\right)$ - is the probability of system failure with $q_{i}=1$.

$Q\left(0_{i}, \mathbf{q}\right)$ - is the probability of system failure with $q_{i}=0$.

Since $\mathrm{Q}_{\text {sys }}$ is a linear function in each $q_{i}$ then $G_{i}(\mathbf{q})$, for each basic event can also be given by:

$$
G_{i}(\mathbf{q})=\frac{\partial Q_{s y s}}{\partial q_{i}}
$$

Evaluating each of the two terms $Q\left(1_{i}, \mathbf{q}\right)$ and $Q\left(0_{i}, \mathbf{q}\right)$ for each component could be achieved by first substituting $q_{i}=1$ and then $q_{i}=0$, i.e., the probability that component $i$ fails is set to 1 and 0 respectively, and re-running all the system failure probability calculations. This would require the equivalent of $2 n$ evaluations of the top event probability where $n$ is the number of components in the system, to deduce all terms required in the expression for $w_{s y s}$ in eq. (9).

However a more efficient way of calculating the criticality function can be achieved directly from the BDD using the formula:

$$
G_{i}(\mathbf{q})=\sum_{x i} p r_{x i}(\mathbf{q})\left[p o_{x i}^{1}(\mathbf{q})-p o_{x i}^{0}(\mathbf{q})\right]
$$

Where:

$\operatorname{pr}_{x i}(\mathbf{q})$ - is the probability of the path section from the root node to node xi

(Probprev).

$p o_{x i}^{1}(\mathbf{q})-$ is the probability of the path section from the 1 branch of node xi to a terminal 1 - node (Probpost 1 branch).

$p o_{x i}^{0}(\mathbf{q})-$ is the probability of the path section from the 0 branch of node $x i$ to a terminal 1 node (Probpost 0 branch).

$n-$ is the number of nodes in the BDD.

Using equation (12) calculating $G_{i}(\mathbf{q})$ requires one pass of the BDD to calculate $p r_{x i}(\mathbf{q})$, $p o_{x i}^{1}(\mathbf{q})$ and $p o_{x i}^{0}(\mathbf{q})$ for each node. With this information each $G_{i}(\mathbf{q})$ can be evaluated from eq. (12) and $w_{s y s}$ formed. 
The algorithm Probpost used to calculate $p o_{x i}^{1}(\mathbf{q})$ and $p o_{x i}^{0}(\mathbf{q})$ is given in figure 3 . The calculation of $p r_{x i}(\mathbf{q})$ can be achieved by the algorithm Probprev given in figure 4 and the criticality function $G_{i}(\mathbf{q})$ for each basic event is calculated as shown in figure 5 .

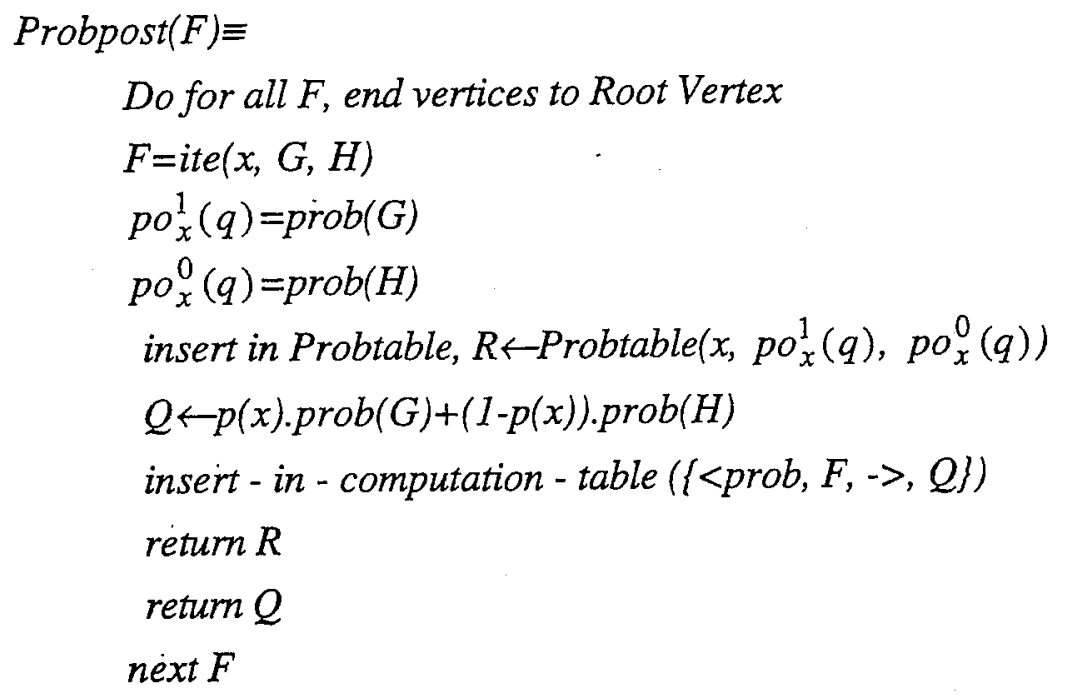

Figure 3. Probpost Algorithm

Set Probprev $(F i)=0$ for all $i$.

$\operatorname{Probprev}(F) \equiv$

start at Root Vertex, $F$

$\operatorname{Probprev}(F)=1$

Add Probprev $(F)$ to Probtable, i.e., Probtable $\left(x, p o_{x}^{1}(q), p o_{x}^{0}(q), p_{x}(q)\right)$

Do for all F, Root Vertex to end vertices

$\mathrm{F}=\mathrm{ite}(\mathrm{x}, \mathrm{H} 1, \mathrm{H} 2)$

if $\mathrm{H} 1=0$ or 1 Goto [A]

$\operatorname{Probprev}(\mathrm{H} 1)=\operatorname{Probprev}(\mathrm{H} 1)+\mathrm{p}(\mathrm{X}) \cdot \operatorname{Probprev}(\mathrm{F})$

Add Probprev(H1) to Probtable

[A] if $\mathrm{H} 2=0$ or 1 next $F$

$\operatorname{Probprev}(\mathrm{H} 2)=\operatorname{Probprev}(\mathrm{H} 2)+(1-\mathrm{p}(\mathrm{x})) \cdot \operatorname{Probprev}(\mathrm{F})$

Add Probprev(H2) to Probtable

next $F$.

Figure 4. Probprev Algorithm 
Set $G(x i)=0$ for all $i$

Do for all $F$

$$
\begin{aligned}
& \text { if } F=\text { Probtable }(x, q 1, q 2, q 3) \\
& G(x)=G(x)+q 3(q 1-q 2) \\
& \text { insert - in Criticality table } G(x)
\end{aligned}
$$

next $F$.

Figure 5. Algorithm for Calculating the Criticality Function $G_{X i}$

The implementation of these algorithms can be demonstrated by their application to the example $\mathrm{BDD}$ given in figure 2. The ite table indicating how the BDD in figure $2 \mathrm{~b}$ is stored within the computer program which performs these calculations is given in table 2 :

\begin{tabular}{|c|ccc|}
\hline Node Label & Variable & 1 branch pointer & 0 branch pointer \\
\hline F1 & X1 & F2 & F4 \\
F2 & X2 & F3 & F4 \\
F3 & X3 & 1 & 0 \\
F4 & X3 & F5 & 0 \\
F5 & X4 & 1 & 0 \\
\hline
\end{tabular}

Table 2. ITE table for the BDD

Performing one pass of the BDD to evaluate $p o_{x i}^{1}(\mathbf{q})$ and $p o_{x i}^{0}(\mathbf{q})$ for each node using Probpost gives:

\section{Probpost(F5).}

$$
\begin{aligned}
& \mathrm{F} 5=\text { ite }(X 4,1,0) \\
& R \leftarrow \operatorname{Probtable}(X 4,1,0) \\
& \mathrm{p} \leftarrow \mathrm{p}(\mathrm{X} 4) \cdot \mathrm{p}(1)+(1-\mathrm{p}(\mathrm{X} 4)) \cdot \mathrm{p}(0)=0.001
\end{aligned}
$$

\section{Probpost(F4)}

$$
\begin{aligned}
& \mathrm{F} 4=\mathrm{ite}(\mathrm{X} 3, \mathrm{~F} 5,0) \\
& \mathrm{R} \leftarrow \operatorname{Probtable}(\mathrm{X} 3, \mathrm{prob}(\mathrm{F} 5), 0) \leftarrow(\mathrm{X} 3,0.001,0) \\
& \mathrm{p} \leftarrow \mathrm{P}(\mathrm{X} 3) .(0.001)+(1-\mathrm{p}(\mathrm{X} 3)) .(0)=2.0 \mathrm{E}-6
\end{aligned}
$$

Probpost(F3)

$$
\begin{aligned}
& \mathrm{F} 3=\mathrm{ite}(\mathrm{X} 3,1,0) \\
& \mathrm{R} \leftarrow \operatorname{Probtable}(\mathrm{X} 3,1,0) \\
& \mathrm{p}-\mathrm{p}(\mathrm{X} 3) \cdot \mathrm{p}(1)+(1-\mathrm{p}(\mathrm{X} 3)) \cdot \mathrm{p}(0)=0.002
\end{aligned}
$$


Probpost(F2)

$\mathrm{F} 2=$ ite $(\mathrm{X} 2, \mathrm{~F} 3, \mathrm{~F} 4)$

$\mathrm{R} \leftarrow$ Probtable $(\mathrm{X} 2$, prob(F3), prob(F4) $\leftarrow(\mathrm{X} 2,0.002,2.0 \mathrm{E}-6)$

$\mathrm{Q}=\mathrm{p}(\mathrm{X} 2) \cdot(0.002)+(1-\mathrm{p}(\mathrm{X} 2)) \cdot(2.0 \mathrm{E}-6)=7.994 \mathrm{E}-6$

Probpost(F1)

$\mathrm{F} 1=\mathrm{ite}(\mathrm{X} 1, \mathrm{~F} 2, \mathrm{~F} 4)$

$\mathrm{R} \leftarrow$ Probtable $(\mathrm{X} 1, \operatorname{prob}(\mathrm{F} 2), \operatorname{prob}(\mathrm{F} 4)) \leftarrow(\mathrm{X} 1,7.994 \mathrm{E}-6,2.0 \mathrm{E}-6)$

$\mathrm{Q}=\mathrm{p}(\mathrm{X} 1) \cdot(7.994 \mathrm{E}-6)+(1-\mathrm{p}(\mathrm{X} 1)) \cdot(2.0 \mathrm{E}-6)=2.023976 \mathrm{E}-6$

As can be seen the probability of the top event, $Q$ (calculated for F1 above) agrees with the probability calculated previously using the disjoint paths of the BDD.

The values of Probpost 1 branch and Probpost 0 branch for each node are entered into the node probability table, PROBTABLE shown in figure 6.

Next Probprev is calculated and entered into the 5th column of the PROBTABLE.

Probprev Algorithm:

$\operatorname{Probprev}(F 1)=\operatorname{Probprev}(F 2)=\operatorname{Probprev}(F 3)=\operatorname{Probprev}(F 4)=\operatorname{Probprev}(F 5)=0$

$\operatorname{Probprev}(\mathrm{F} 1)=1$

$\mathrm{F} 1=\mathrm{ite}(\mathrm{X} 1, \mathrm{~F} 2, \mathrm{~F} 4)$

Probprev $(\mathrm{F} 2)=0+\mathrm{p}(\mathrm{X} 1) \cdot \operatorname{Probprev}(\mathrm{F} 1)$ $=(0.004) .(1)=0.004$

Probprev(F4) $=0+(1-\mathrm{p}(\mathrm{X} 1)) \cdot \operatorname{Probprev}(\mathrm{F} 1)$

$=(1-0.004) \cdot(1)=0.996$

$F 2=i t e(X 2, F 3, F 4)$

$\operatorname{Probprev}(\mathrm{F} 3)=0+\mathrm{p}(\mathrm{X} 2) \cdot \operatorname{Probprev}(\mathrm{F} 2)$

$=(0.003) \cdot(0.004)=1.2 \mathrm{E}-5$

Probprev $(F 4)=0.996+(1-p(X 2)) \cdot$ Probprev $(F 2)$

$=0.996+(1-0.003) \cdot(0.004)=0.999988$ 


$$
\begin{gathered}
F 3=\operatorname{ite}(X 3,1,0) \\
H 1=1 \\
H 2=0
\end{gathered}
$$

$$
\begin{aligned}
\mathrm{F} 4=\mathrm{ite}(\mathrm{X} 3, \mathrm{~F} 5,0) & \\
\text { Probprev }(\mathrm{F} 5) & =0+\mathrm{p}(\mathrm{X} 3) \cdot \operatorname{Probprev}(\mathrm{F} 4) \\
& =(0.002) \cdot(0.999988)=1.999976 \mathrm{E}-3 \\
\mathrm{H} 2=0 &
\end{aligned}
$$

$$
\begin{gathered}
\text { F5 }=\text { ite }(X 4,1,0) \\
H 1=1 \\
H 2=0
\end{gathered}
$$

PROBTABLE

\begin{tabular}{|c|cccc|}
\hline Node Label & Variable & post '1' & post '0' & Probprev \\
\hline F1 & X1 & $7.994 \mathrm{E}-6$ & $2.0 \mathrm{E}-6$ & 1 \\
F2 & $\mathrm{X} 2$ & 0.002 & $2.0 \mathrm{E}-6$ & 0.004 \\
F3 & $\mathrm{X} 3$ & 1 & 0 & $1.2 \mathrm{E}-5$ \\
F4 & $\mathrm{X} 3$ & 0.001 & 0 & 0.999988 \\
F5 & $\mathrm{X} 4$ & 1 & 0 & $1.999976 \mathrm{E}-3$ \\
\hline
\end{tabular}

Probtable $(i, 1)=$ Node Label

Probtable $(i, 2)=$ Basic event of node Fi

Probtable( $(\mathrm{i}, 3)=$ Probability of post ' 1 ' branch

Probtable $(i, 4)=$ Probability of post ' 0 ' branch

Probtable $(i, 5)=$ Probability of previous

Figure 6. PROBTABLE Array

Calculation of the criticality function is then straight forward using the algorithm provided in figure 5. The following values of the criticality function for each basic event are obtained.

$\mathrm{G}_{\mathrm{X} 1}=5.994 \mathrm{E}-6, \quad \mathrm{G}_{\mathrm{X} 2}=7.992 \mathrm{E}-6, \quad \mathrm{G}_{\mathrm{X} 3}=1.011988 \mathrm{E}-3, \quad \mathrm{G}_{\mathrm{X} 4}=1.999976 \mathrm{E}-3$

Since we have calculated the criticality function for each component, the system parameter $w_{\text {sys }}$ can now be evaluated using the basic event frequency data from table 1 and eq. (9).

$$
\begin{aligned}
w_{s y s} & =G_{X 1} w_{X 1}+G_{X 2} w_{X 2}+G_{X 3} w_{X 3}+G_{X 4} w_{X 4} \\
& =1.1391734 \mathrm{E}-11
\end{aligned}
$$


The expected number of top event occurrences in time, $t$, can be obtained from eq. (8).

\section{Importance Measures}

A very useful piece of information which can be derived from a fault tree study is the importance measure for each component or each minimal cut set. An importance analysis provides sensitivity measures which identifies weak areas of the system. This information can be very valuable particularly at the design stage. For each component its importance signifies the role that it plays in either causing or contributing to the occurrence of the top event. In general a numerical value is assigned to each basic event or minimal cut set which allows it to be ranked along with other failure events according to the extent of its contribution to the occurrence of the top event.

Probabilistic importance measures can be categorised in two ways: (i) those which are appropriate for system availability assessment (top event probability) and (ii) those which are concerned with system reliability assessment (expected number of top event occurrences), here we are concerned only with component measures in the first group.

\section{(i) Birnbaum Measure of Component Importance}

The Birnbaum Measure of importance $\left(I_{b}\right)$ was first introduced in 1969 (Ref. 8). This measure is defined as the rate at which the system failure probability changes as the failure probability of component i changes:

$$
I_{b i}=\frac{\partial Q_{s y s}}{\partial q_{i}}
$$

As defined in eq. (11) $I_{b i}$ is also the criticality function for component $i, G_{i}(q)$. The calculation procedure to evaluate the criticality function from the BDD structure has been described previously. Hence the calculation of this importance measure for each component is performed in the calculation of $w_{s y s}$ and can therefore be extracted from those calculations.

\section{(ii) Criticality Measure of Component Importance}

The criticality measure of importance $\left(I_{c}\right)$ is defined as:

"The probability that the system is in a state at time $t$ which is critical for component $i$ and that component $i$ has failed at time $t$ conditional on system failure at time $t . "$ 


$$
\begin{aligned}
I_{c i} & =\frac{\left\{Q\left(1_{i}, \mathbf{q}\right)-Q\left(0_{i}, \mathbf{q}\right)\right\} q_{i}}{Q(\mathbf{q})} \\
& =\frac{G_{i}(\mathbf{q}) \cdot q_{i}}{Q_{s y s}}
\end{aligned}
$$

All terms in eq. (14) have been evaluated in calculating $Q_{s y s}$ and $w_{\text {sys }}$ and are readily available to determine this importance measure.

\section{(iii) Fussell-Vesely Measure of Component Importance}

This measure of Importance is usually close in numerical value to the criticality measure. The Fussell-Vesely Importance $\left(I_{F V}\right)$ is defined as the probability of the Union of the Minimal Cut Sets which contain event i divided by the top event occurrence probability. $I_{F V}$ therefore gives the probability that when the system fails, component $i$ contributed to the failure. Calculating $I_{F V}$ for each component requires the use of both the minimal BDD to track the minimal cut sets and the non-minimal BDD for the probability calculations. The calculation of $I_{F V}$ is performed in 4 steps:

(1) First a counter for the number of times each variable is encountered in a minimal cut set is initialised.

(2) A search is then performed on each path of the minimum BDD which will correspond to a minimal cut set. For each variable in the path i.e. the path passes out of the 1 branch, the occurrence counter is incremented. Once the search has been completed, it is known how many minimal cut sets contain each basic event.

(3) For events with more than one occurrence a search is made for the nodes in the nonminimal $\mathrm{BDD}$ that have this variable. For each basic event:

$$
I_{F V i}=\frac{\sum_{x i \text { nodes }} q_{i} p r_{x i}(\mathbf{q}) p o_{x i}^{1}(\mathbf{q})}{Q_{s y s}}
$$

(4) If a variable has just one occurrence in a minimal cut set, $c_{i}$ then:

$$
I_{F V i}=\frac{p\left(c_{i}\right)}{Q_{s y s}}
$$




\section{Applications}

The BDD quantification method was benchmarked against a test example fault tree called 'Dresden-3' used by Platz and Olsen (Ref. 9). The structure file and data file for this tree are contained in appendix A. The input file gives the gate name (a number over 1000), the gate type, the number of gate inputs, the number of basic event inputs, followed by a list of the inputs themselves. Each basic event in the fault tree has two lines of data to specify its failure and repair characteristics, the first line gives the name of the basic event and the model type, in this case model type $F$ was used for each component. The second line gives, for this failure model, parameters, $\lambda$ and $\tau$ ( $\lambda$ must be multiplied by $1 \mathrm{E}-6$ ). $\lambda$ represents the component constant failure rate and $\tau$ its mean time to repair (MTTR)

The following calculations are then used for the model type $F$, to obtain $q_{i}$ and $w_{i}$ for each basic event.

$$
\begin{aligned}
& q_{i}=\lambda . \tau /(\lambda . \tau+1) \\
& w_{i}=\lambda\left(1-q_{i}\right)
\end{aligned}
$$

A summary of the quantification results is given in table 3. The code runs on a Sun workstation, the execution time is given in seconds.

\begin{tabular}{|c|c|}
\hline Name & Dresden-3 \\
\hline No. of Gates & 60 \\
\hline No. of Basic Events & 57 \\
\hline No. of Minimal Cut Sets & 11,934 \\
\hline Time (s) & 0.6 \\
\hline$Q_{\text {sys }}$ & $4.70085 \mathrm{E}-7$ \\
\hline$w_{\text {sys }}$ & $2.87887 \mathrm{E}-8$ \\
\hline
\end{tabular}

Table 3. BDD quantification results for Dresden-3 fault tree

As a comparison, Dresden-3 was analysed using a state-of-the art conventional Fault Tree Analysis package, whose results can be seen in table 4 .

\begin{tabular}{|c|c|}
\hline No. of Minimal Cut Sets & 11,934 \\
\hline Time & 4hrs $10 \mathrm{~min} 28 \mathrm{~s}$ \\
\hline$Q_{\text {sys }}$ & $4.81119 \mathrm{E}-7$ \\
\hline$w_{s y s}$ & $2.97304 \mathrm{E}-8$ \\
\hline
\end{tabular}

Table 4. FTA quantification of Dresden-3 fault tree 
Hence, for this example the BDD method is significantly faster than conventional quantification techniques. Also, along with great savings in computation time the BDD technique gives exact probability values for $Q_{s y s}$ and $w_{s y s}$, whereas the conventional method results in a loss in accuracy of $2.34 \%$ and $3.27 \%$ respectively for these parameters.

\section{Accuracy - Comparison with FTA}

To compare the accuracy of the BDD technique with the conventional Kinetic Tree theory approach, 10 example fault trees were analysed, the results of which are given in table 5 . Some of these benchmark fault trees are taken from industry and the others are produced as simple structures to test different aspects of the analysis code.

\begin{tabular}{|c|c|c|c|c|c|c|c|}
\hline Tree & $\begin{array}{c}\text { No. of } \\
\text { Gates }\end{array}$ & $\begin{array}{c}\text { No. of } \\
\text { basic } \\
\text { events }\end{array}$ & $\begin{array}{c}\text { No. of } \\
\text { Minimal } \\
\text { Cut Sets }\end{array}$ & $\begin{array}{c}\text { BDD } \\
Q_{s y s}\end{array}$ & $\begin{array}{c}\text { FTA } \\
Q_{s y s}\end{array}$ & $\begin{array}{c}\text { B DD } \\
w_{\text {sys }}\end{array}$ & $\begin{array}{c}\text { FTA } \\
w_{\text {sys }}\end{array}$ \\
\hline 1 & 17 & 11 & 43 & $2.08587 \mathrm{E}-2$ & 0.0209883 & $7.52376 \mathrm{E}-6$ & $8.03221 \mathrm{E}-6$ \\
2 & 63 & 32 & 8,716 & $4.272258 \mathrm{E}-7$ & $4.27248 \mathrm{E}-7$ & $2.777932 \mathrm{E}-4$ & 0.000277835 \\
3 & 21 & 40 & 416 & $1.317774 \mathrm{E}-6$ & $1.31778 \mathrm{E}-6$ & $8.990849 \mathrm{E}-4$ & 0.0008991 \\
4 & 10 & 10 & 13 & $6.43795 \mathrm{E}-2$ & 0.068559 & $1.96564 \mathrm{E}-4$ & 0.000211151 \\
5 & 4 & 6 & 3 & $3.39397 \mathrm{E}-8$ & $3.4 \mathrm{E}-8$ & $2.74191 \mathrm{E}-10$ & $2.7474 \mathrm{E}-10$ \\
6 & 4 & 6 & 6 & $7.06927 \mathrm{E}-5$ & $7.10911 \mathrm{E}-5$ & $3.09498 \mathrm{E}-6$ & $3.12839 \mathrm{E}-6$ \\
7 & 3 & 4 & 2 & $3.0776 \mathrm{E}-4$ & 0.000308 & $6.07692 \mathrm{E}-7$ & $6.0836 \mathrm{E}-7$ \\
8 & 10 & 8 & 10 & $1.23233 \mathrm{E}-5$ & $1.2371 \mathrm{E}-5$ & $2.27332 \mathrm{E}-7$ & $2.28347 \mathrm{E}-7$ \\
9 & 3 & 4 & 2 & $2.02398 \mathrm{E}-6$ & $2.02656 \mathrm{E}-6$ & $1.13917 \mathrm{E}-11$ & $1.1392 \mathrm{E}-11$ \\
10 & 30 & 60 & 7,056 & $4.371846 \mathrm{E}-7$ & $4.17233 \mathrm{E}-7$ & $3.092103 \mathrm{E}-4$ & 0.000297212 \\
\hline
\end{tabular}

Table 5. Quantification Results of 10 example fault trees

It is evident from the results in table 4 that when the fault tree has a small number of minimal cut sets the conventional approximation method has only a small error - an average over estimate of $1.22 \%$ for the system failure probability and $2.3 \%$ for the system unconditional failure intensity. However, for tree 10 , which has a large number of minimal cut sets, there is an error of $4.56 \%$ for $Q_{s y s}$ and $3.88 \%$ for $w_{s y s}$. These inaccuracies should be avoided when a risk assessment is performed on an industrial system. 


\section{Conclusion}

The use of BDDs to improve the efficiency of calculating the minimal cut sets and prime implicants of a fault tree has been proven for large complex fault trees by Rauzy Ref. (1) and Ref. (4).

This paper extends the use of the BDD method to calculate top event parameters, such as system failure probability, failure intensity and expected number of top event occurrences. The added advantage of obtaining these parameters directly from the BDD, when compared to traditional Kinetic tree theory approach (Ref. (10) and Ref. (11)), is that the resulting values are exact. Approximations used in conventional fault tree analysis are shown to be inadequate for some fault trees.

The paper has also shown that the commonly used component Importance measures for top event probability can be calculated directly from the BDD. Further, the BDD method has proven to be extremely efficient as a means of quantification. Only one pass of the BDD structure is required to calculate all parameters.

\section{References}

1. A. Rauzy, "New algorithms for fault tree analysis," Reliability Engineering and System Safety, vol 40, 1993, pp203-211.

2. W.G. Schneeweiss, "Boolean Functions with Engineering Applications and Computer Programs," Springer-Verlag, 1989.

3. R.M. Sinnamon and J.D. Andrews, "New Approaches to Evaluating Fault Trees," Proceedings of Esrel'95 Conference, June, 1995 pp241-254.

4. A. Rauzy et al., 'Computation of prime implicants of a fault tree within Aralia," Proceedings of Esrel'95 Conference, June, 1995 pp190-202.

5. R.M. Sinnamon and J.D. Andrews, "Improved Efficiency in Qualitative Fault Tree Analysis", 12th Arts, Advances in Reliability Technology Symposium, Manchester, April 1996.

6. J.D. Andrews and T.R. Moss, "Reliability and Risk Assessment," Longman Scientific and Technical, 1993. 
7. E.J. Henley and H. Kumamoto, "Reliability Engineering and Risk Assessment," Englewood Cliffs, 1981.

8. Z.W. Birnbaum, "On the Importance of Different Components in a Multicomponent System", in Multivariate Analysis II, P. R. Krishnaiah (Ed), Academic Press, 1969.

9. O. Platz and J.V. Olsen, "FAUNET: A Program Package for Evaluation of Fault Trees and Network," Research Establishment, Ris $\varnothing$ Report No.348, DK-4000 Roskilde, Denmark, Sept. 1976.

10. J.B. Fussell and W. E. Vesely, "A new methodology for obtaining cut sets for fault trees", Trans Am Nucl Soc, Vol 15, 1972, June, pp262-263

11. W.E. Vesely, "A Time Dependent Methodology for Fault Tree Evaluation", Nuclear Eng and Des, 13, 1970, pp337-360. 


\begin{tabular}{|c|c|c|c|c|c|c|}
\hline 1060 & & and & 2 & 01058 & 1059 & \\
\hline 1058 & & and & 2 & 01057 & 1055 & \\
\hline 1059 & & and & 2 & 01053 & 1045 & \\
\hline 1057 & & and & 3 & 01037 & 1034 & 1032 \\
\hline 1055 & & and & 3 & 01026 & 1022 & 1019 \\
\hline 1053 & . & or & 2 & 01052 & 1051 & \\
\hline 1045 & & or & 2 & 01044 & 1043 & \\
\hline 1044 & & or & 0 & 249 & 48 & \\
\hline 1043 & & or & 1 & 11042 & 47 & \\
\hline 1052 & & or & 0 & 257 & 56 & \\
\hline 1051 & & or & 1 & 11050 & 55 & \\
\hline 1042 & & or & 2 & 01041 & 1040 & \\
\hline 1050 & & or & 2 & 01049 & 1048 & \\
\hline 1049 & & or & 0 & 254 & 53 & \\
\hline 1048 & & or & 1 & 11047 & 52 & \\
\hline 1041 & & or & 0 & 246 & 45 & \\
\hline 1040 & & or & 1 & 11039 & 44 & \\
\hline 1047 & & or & 2 & 01057 & 1046 & \\
\hline 1039 & & or & 2 & 01055 & 1038 & \\
\hline 1046 & & or & 0 & 250 & 51 & \\
\hline 1038 & & or & 0 & 243 & 42 & \\
\hline 1037 & & or & 2 & 01036 & 1025 & \\
\hline 1034 & & or & 2 & 01033 & 1020 & \\
\hline 1032 & & or & 2 & 01056 & 1031 & \\
\hline 1036 & & or & 0 & 241 & 40 & \\
\hline 1025 & & or & 2 & 11024 & 1035 & 29 \\
\hline 1033 & - & or & 0 & 237 & 36 & \\
\hline 1020 & & or & 0 & 224 & 23 & \\
\hline 1056 & & and & 2 & 01030 & 1028 & \\
\hline 1031 & & or & 0 & 235 & 34 & \\
\hline 1024 & & or & 0 & 228 & 27 & \\
\hline 1035 & & or & 0 & 239 & 38 & \\
\hline 1030 & & or & 2 & 01029 & 1009 & \\
\hline 1028 & & or & 2 & 01027 & 1017 & \\
\hline 1029 & & or & 0 & 233 & 32 & \\
\hline 1009 & & or & $I$ & 11008 & 10 & \\
\hline 1027 & & or & 0 & 231 & 30 & \\
\hline 1017 & & or & 1 & 11015 & 18 & \\
\hline 1015 & & or & 1 & 11014 & 16 & \\
\hline 1008 & & or & 2 & 01007 & 1006 & \\
\hline 1014 & & or & 2 & 01013 & 1012 & \\
\hline 1007 & & or & 0 & 29 & 8 & \\
\hline 1006 & & or & 0 & 27 & 2 & \\
\hline 1013 & & or & 0 & 215 & 14 & \\
\hline 1012 & & or & 1 & 11005 & 13 & \\
\hline 1005 & & or & 2 & 01004 & 1003 & \\
\hline 1004 & & or & 0 & 26 & 5 & \\
\hline 1003 & & or & 2 & 01002 & 1001 & \\
\hline 1002 & & or & 0 & 24 & 3 & \\
\hline 1001 & & or & 0 & 22 & 1 & \\
\hline 1026 & & or & 2 & 01025 & 1023 & \\
\hline 1022 & & or & 2 & 01021 & 1020 & \\
\hline 1019 & & or & 2 & 01054 & 1018 & \\
\hline 1023 & & or & 0 & 226 & 25 & \\
\hline 1021 & & or & 0 & 222 & 21 & \\
\hline 1054 & & and & 2 & 01016 & 1011 & \\
\hline 1018 & & or & 0 & 220 & 19 & \\
\hline 1016 & & or & 1 & 11015 & 17 & \\
\hline 1011 & & or & 1 & 11010 & 12 & \\
\hline 1010 & & or & 1 & 11009 & 11 & \\
\hline
\end{tabular}


Datizi file for Dresden-?

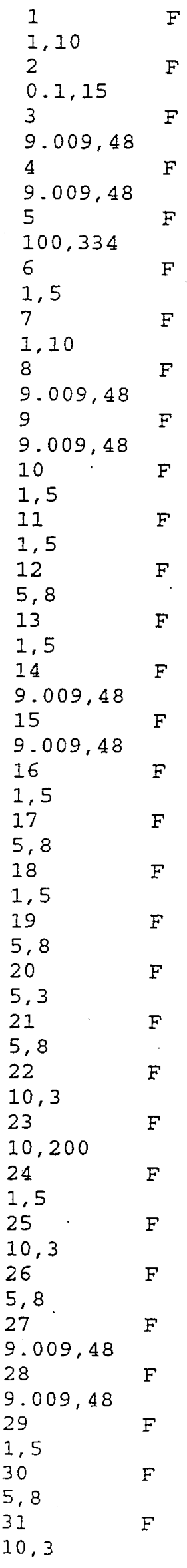

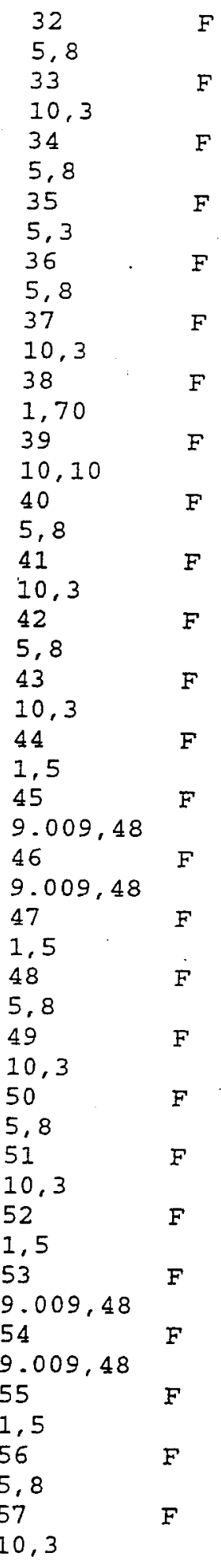

